\title{
ON TWO QUESTIONS OF MOORE AND MROWKA
}

\author{
S. P. FRANKLIN
}

0. Introduction. Let $X$ be a $T_{1}$-space. In a 1964 Abstract [3], Moore and Mrowka consider the following three properties:

(1) any subset $A$ of $X$, which contains the limits of all sequences from $A$, is closed;

(2) any subset of $X$, which contains the closure of each of its subsets with countable closures, is closed; and

(3) any subset of $X$, which contains the closure of each of its countable subsets, is closed.

Spaces satisfying (1) are precisely the sequential spaces [1], [2]. Those satisfying (2) are said to be determined by countable closed subsets. Those satisfying (3) are the c-spaces [5]. Moore and Mrowka note that (2) always implies (3), that for Hausdorff spaces (1) implies (2), and that for compact Hausdorff spaces (1) and (2) are equivalent. They ask if there is a Hausdorff space satisfying (3) but not (2), and if there is a compact Hausdorff space satisfying (3) but not (1). In this note we give a complete answer to the first question and a partial answer to the second as follows:

THEOREM A. There is a totally disconnected functionally Hausdorff c-space which is not determined by countable closed sets.

THEOREM B. Every compact Hausdorff c-space of cardinality $<2^{\aleph_{1}}$ is sequential.

Note that such a space need not be first countable. (Take for example the one point compactification of a discrete space of cardinality $\boldsymbol{\aleph}_{1}$.)

The new questions arising from these results are:

(a) Is there a regular $c$-space which is not determined by closed countable subsets?

(b) Given the continuum hypothesis, is there a separable compact Hausdorff $c$-space of cardinality $2^{c}$ which is not sequential?

An answer to question (b) together with Theorem B would settle the matter, for suppose $X$ is any compact Hausdorff $c$-space which is not sequential. Then there is a sequentially closed subset $F$ of $X$ which is not closed. Choose $x \in \operatorname{cl}_{X} F \backslash F$. There is some countable subset $C$ of $F$ with $x \in \operatorname{cl}_{X} C$. Then $\mathrm{cl}_{X} C$ is a separable compact Hausdorff

Received by the editors May 17, 1968. 
$c$-space which is not sequential. By Theorem B and the continuum hypothesis its cardinality is $2^{c}$. Thus the general case reduces to that of question $b$.

1. Proof of Theorem $\mathbf{A}$. As usual, let $\mathbf{N}$ be the natural numbers and $\beta N$ its Stone-Cech compactification. Let $F$ be $\beta N$ retopologized with the smallest topology making $\beta \boldsymbol{N} \backslash \boldsymbol{N}$ a discrete subset. (The open sets may be described as follows: For $p \in \beta N \backslash N$, let $N_{p}=\{p\} \cup N$. The new topology is generated by the union of the old topology and the family $\left\{N_{p} \mid p \in \beta N \backslash N\right\}$.) Since both properties are preserved under increases in the topology, $F$ is totally disconnected and functionally Hausdorff. Now $A$ is closed in $F$ if and only if $\operatorname{cl}_{\beta N}(A \cap N) \subseteq A$, since $A \cup \operatorname{cl}_{\beta \boldsymbol{N}}(A \cap N)$ is the closure of $A$ in $F$. Hence $F$ is a $c$-space. Since only finite subsets of $N$ have countable closures in $F$ but $N$ is not closed in $F, F$ is not determined by countable closed subsets. Also note that no $p \in \beta \boldsymbol{N} \backslash \boldsymbol{N}$ can be separated from the closed set $F \backslash \boldsymbol{N}_{p}$ by disjoint open sets. Hence $F$ is not regular and in particular is not zero dimensional.

(I am indebted to the referee for an improvement of the original construction of this example.)

2. Proof of Theorem B. We shall prove a stronger version of Theorem B which is a corollary of a corollary to a theorem of Mrowka [4].

MROWKA's THEOREM. Every uncountable compact Hausdorff space of cardinality $\left\langle 2 \aleph^{\aleph_{1}}\right.$ has uncountably many points of first countability (i.e. of countable local weight).

From this theorem it is easy to prove the following:

COROLLARY. Every compact Hausdorff space of cardinality $<2 \aleph_{1}$ is sequentially compact.

Indeed, the closure of a sequence of distinct points in such a space is compact Hausdorff and hence, if countable, is first countable and therefore sequentially compact. If the closure is uncountable, by Mrowka's Theorem there is a point of first countability outside the dense sequence. Every such point is the limit of a subsequence.

We need only one more preliminary.

Proposition. Every locally sequentially compact Hausdorff c-space is sequential.

Indeed, let $X$ be such a space and $F$ one of its sequentially closed subsets. If $x_{0} \in \operatorname{cl}_{X} F$, some sequence $\left\{x_{n}\right\}$ in $F$ clusters at $x_{0}$. Let $K$ 
be a sequentially compact neighborhood of $x_{0}$. Then some subsequence of $\left\{x_{n}\right\}$ lies in $K$ and hence some subsequence converges to $x_{0}$ (since $X$ is Hausdorff). Thus $x_{0} \in F$ and $X$ is sequential.

Combining the Corollary and the Proposition yields the promised strong form of Theorem B.

THEOREM C. Every locally compact Hausdorff c-space of local cardinality $<2 \aleph_{1}$ is sequential.

It follows from the Corollary and the Proposition that such a space is locally sequential and hence is sequential $[2, \mathrm{p} .53]$.

\section{REFERENCES}

1. S. P. Franklin, Spaces in which sequences suffice, Fund. Math. 57 (1965), $107-115$.

2. - Spaces in which sequences suffice. II, Fund. Math. 61 (1967), 51-56.

3. R. C. Moore and G. S. Mrowka, Topologies determined by countable objects, Notices Amer. Math. Soc. 11 (1964), 554.

4. S. Mrowka, On the potency of compact spaces, Bull. Acad. Polon. Sci. Sér. Sci. Math. Astronom. Phys. 6 (1958), 7-9.

5. T. W. Richel, $A$ class of spaces determined by sequences with their cluster points, Notices Amer. Math. Soc. 14 (1967), 698-699.

INDIAN INSTITUTE OF TECHNOLOGY, KaNPUR AND

Carnegie-Mellon University 